\title{
Computerised cognitive-behavioural therapy for adults with intellectual disability: randomised controlled trial
}

\author{
Patricia Cooney, Catherine Jackman, David Coyle and Gary O'Reilly
}

\section{Background}

Despite the evidence base for computer-assisted cognitivebehavioural therapy (CBT) in the general population, it has not yet been adapted for use with adults who have an intellectual disability.

\author{
Aims \\ To evaluate the utility of a CBT computer game for adults \\ who have an intellectual disability.
}

\section{Method}

A $2 \times 3$ (group $\times$ time) randomised controlled trial design was used. Fifty-two adults with mild to moderate intellectual disability and anxiety or depression were randomly allocated to two groups: computerised CBT (CCBT) or psychiatric treatment as usual (TAU), and assessed at pre-treatment, post-treatment and 3-month follow-up. Forty-nine participants were included in the final analysis.

\section{Results}

A significant group $\times$ time interaction was observed on the primary outcome measure of anxiety (Glasgow Anxiety Scale for people with an Intellectual Disability), favouring CCBT over
TAU, but not on the primary outcome measure of depression (Glasgow Depression scale for people with a Learning Disability). A medium effect size for anxiety symptoms was observed at post-treatment and a large effect size was observed after follow-up. Reliability of Change Indices indicated that the intervention produced clinically significant change in the CCBT group in comparison with TAU.

\section{Conclusions}

As the first application of cCBT for adults with intellectual disability, this intervention appears to be a useful treatment option to reduce anxiety symptoms in this population.

\section{Declaration of interest}

P.C., C.J., D.C. and G.O.'R. designed the computerised CBT program, Pesky Gnats: The Feel Good Island, used in the present study. None of the authors receives any income from the program, which is owned by Handaxe, a community interest company run on a not-for-profit basis.

\section{Copyright and usage}

(c) The Royal College of Psychiatrists 2017.
Prevalence rates of mental health problems in adults who have an intellectual disability are reported to range from $15.7 \%$ to $40.9 \%$ depending on diagnostic criteria used. ${ }^{1}$ Looking to prevalence rates in the general population, it is clear that people who have an intellectual disability experience a similar, if not greater, prevalence of mental health problems than the general population. ${ }^{2}$ Still, the establishment of evidenced-based psychological therapies has been slow in comparison with the general population. Recently there have been considerable efforts in this field to increase the provision of evidence-based psychological therapies for adults who have an intellectual disability. A recent meta-analysis by Koslowski et al $^{3}$ examined the effectiveness of existing interventions in treating mental health problems in adults with a mild to moderate intellectual disability. Findings reported that no type of intervention (psychotherapeutic, biological and system level) obtained a statistically significant effect size for the treatment of depression or anxiety. The most encouraging but modest finding was that CBT appeared to be a promising treatment for depression and thus warrants further research. Furthermore, recent National Institute for Health and Care Excellence (NICE) ${ }^{4}$ guidelines recommend CBT for depression and subthreshold depressive symptoms, and relaxation therapy and graded exposure to treat anxiety in this population. Fewer trials evaluating CBT for the treatment of anxiety exist for this population, and for the most recent controlled trials the outcomes appear to be weak. ${ }^{5-7}$ Computerised CBT (cCBT) is a method of delivering the core principles of cognitive and behavioural approaches through a computer or tablet at home or in a healthcare setting, online only or with telephone support from a practitioner. $\mathrm{NICE}^{8}$ guidelines recommend $\mathrm{CCBT}$ as a treatment for persistent subthreshold, mild to moderate depression in adults in the general population. Despite the evidence base for CCBT in the general population, it has not yet been developed specifically for use with people who have an intellectual disability as far as the authors are aware. A number of technology-assisted interventions have been developed for people who have an intellectual disability to target academic, communication, employment and leisure skills, and most recently to teach CBT skills that assist engagement in CBT. ${ }^{9,10}$ It must be acknowledged that although there has been recent progress in technology acquisition and use by people with an intellectual disability, a technological divide still exists whereby technologies are underutilised in adults with intellectual disability in comparison with the general population. ${ }^{11}$

Currently, there is a critical need to develop evidence-based psychological interventions to meet the mental health needs of adults who have an intellectual disability. The present study aims to contribute to this area by developing and evaluating the utility of a prototype CBT computer game, Pesky Gnats: The Feel Good Island, ${ }^{12}$ as a novel psychological intervention to treat anxiety and depression in a sample of adults who have a mild to moderate intellectual disability.

\section{Method}

\section{Trial design}

A $2 \times 3$ (group $\times$ time) randomised controlled trial $(\mathrm{RCT})$ design was used in the present study. Fifty-two participants were randomly allocated to two groups: the CCBT group who received a 7-week CBT computer game intervention and the TAU group who continued to receive routine psychiatric treatment. Both 
groups were assessed with primary and secondary outcome measures at pre-treatment (Time 1), post-treatment at 7 weeks (Time 2), and 3-month follow-up treatment (Time 3). Ethical approval was obtained from the host university's ethics committee (HS-13-58-Cooney-OReilly).

\section{Participants}

A sample of 52 adults was recruited from one voluntary organisation providing residential and day services to children and adults with an intellectual disability. The catchment area of the centre and its community services is the Health Services Executive Dublin South West Region. The centre's multidisciplinary team includes medical services (psychiatry, general practice service, paediatrician and pharmacist), social work, psychology, occupational therapy, speech and language therapy, music therapy, physiotherapy, and a dietician. The Mental Health of Intellectual Disability (MHID) team is a specialist secondary care service in this centre which provides non-crisis mental health services for adults aged 18 years and over who have an intellectual disability and mental health needs. The service's clinical case-load was 104 people when participants were recruited for the present study. Eligibility criteria for participants to take part in the present study were: 18 years or older, an Axis I diagnosis of a mild or moderate intellectual disability, an Axis II diagnosis of anxiety, depression, comorbid anxiety and depression, or recurring anxiety or depression. Exclusion criteria for participants were: below age 18 years, an Axis I diagnosis of a severe to profound intellectual disability, an Axis II diagnosis of bipolar disorder, schizophrenia or other psychotic disorder, selective mutism, or if the participant was non-verbal or considered to be too unwell to engage in psychological therapy. Four people who were involved in the development of the intervention were excluded from the present study. These people were offered the intervention once the present study had finished. The principal clinical psychologist (C.J.) of the service carried out the intervention and collected data from the cCBT group at each time point. The primary researcher (P.C.) collected data from the TAU group. Data were collected onsite in the main centre, within the participant's onsite residence, or in offsite community day-service centres.

Demographic and clinical characteristics of the cCBT group and the TAU group are presented in Table 1 (see online Table DS1 for complete results). Comparisons of the main demographic and clinical characteristics between the cCBT and TAU groups were carried out using independent $t$-tests and $\chi^{2}$-tests for continuous and categorical variables respectively; $\chi^{2}$-tests were not computed for variables which contained cells with an expected count less than 5 . There were no differences between both groups on any variable.

\section{Intervention}

Pesky Gnats: The Feel Good Island is an adapted version of the original Pesky gNATs CBT computer game ${ }^{13}$ and mobile application $(\mathrm{app})^{14}$ for young people aged $9-16$ years who experience clinically significant anxiety or low mood as previously developed by our team. Pesky gNATs is part of a range of computer games and apps our team is designing and releasing on a not-for-profit basis that combine technology and evidence-based psychological research in mental health, details of which can be found on our website http:// peskygnats.com. At its current stage of development, Pesky Gnats: The Feel Good Island is a prototype computer game which we are presently evaluating with the aim of making future versions available on a sustainable not-for-profit basis.

Pesky Gnats: The Feel Good Island is a CBT computer game that teaches an adult who has an intellectual disability and is experiencing clinically significant anxiety or depression how to manage their mood by employing the general cognitive model. ${ }^{15}$ The game simplifies core CBT concepts into a meaningful social story that makes understanding the cognitive model easier for adults with an intellectual disability. The computer game was played by an adult alongside the clinical psychologist for seven consecutive weekly sessions. Therapy sessions lasted approximately $1 \mathrm{~h}$. As the player explores the 3D game world they meet six different game characters who teach them seven core CBT skills across seven game levels (online Fig. DS1). The player completes a mindfulness or relaxation exercise presented as a video with audio at the end of each session (Fig. DS1). Players complete between-therapy skills practice using the Pesky Gnats: Feel Good Island workbook. The workbook is divided into seven chapters reflecting each level of the game. It provides a largely visual reminder of the content of each therapy session designed to support the person completing the intervention to implement $\mathrm{CBT}$, mindfulness, and relaxation skills in their daily life at home, vocational setting or community. Participants could record their use of the workbook in writing or by using a set of visual stickers depending on their preference or level of ability.

Participants in the TAU group continued to receive ongoing psychiatric treatment. Psychiatric treatment as usual for this group involved dietician input, weight management and epilepsy management provided by recognised specialist national programmes, and additional in-home, employment and community supports. Forty per cent of the TAU group were receiving pharmacological treatment for anxiety and mood disorders. Ongoing psychological input provided to the TAU group by a clinical psychologist included weekly bereavement counselling for one participant, weekly individual CBT for anger management for one participant, and weekly brief focused CBT for one participant. One participant in the cCBT group continued to receive counselling from a clinical psychologist during the study.

\section{Objectives}

The aim of this study was to establish the utility of a CBT computer game for adults who have anxiety or depression and mild or moderate intellectual disability. The objective was to investigate this aim using an RCT design. The main hypothesis of the present study was: can Pesky Gnats: The Feel Good Island CBT computer game significantly reduce levels of anxiety and depression in adults who have a mild or moderate intellectual disability?

\section{Outcomes}

\section{Primary outcome measures}

The Glasgow Anxiety Scale for people with an Intellectual Disability $(\text { GAS-ID })^{16}$ is a self-report measure used to assess state anxiety. It has three subscales: worries, specific fears and physiological symptoms. Mindham \& Espie ${ }^{16}$ suggest that a cut-off score of 13 and above may be used to identify a possible anxiety disorder. The total scale has high test-retest reliability of $r=0.95$, high internal consistency reliability of $\alpha=0.96$ and $\alpha=0.87$ in the present sample. $^{16}$

The Glasgow Depression Scale for people with a Learning Disability (GDS-LD) ${ }^{17}$ is a self-report measure used to assess 'present state' depression symptoms. Cuthill et al ${ }^{17}$ recommend a cut-off score of 13 to detect individuals with depression. The GDS-LD has high test-retest reliability of $r=0.95$ and high internal consistency reliability of $\alpha=0.90$ and $\alpha=0.82$ in the present sample. ${ }^{17}$

\section{Secondary outcome measure}

The Clinical Outcomes in Routine Evaluation - Learning Disability $(\text { CORE-LD })^{18}$ is a self-report measure assessing psychological 


\begin{tabular}{|c|c|c|c|}
\hline & CCBT group $(n=24)$ & TAU group $(n=25)$ & Statistic $^{a, b}$ \\
\hline Age, years: mean (s.d.) range & $42.00(12.85) 23-69$ & $39.24(9.14) 25-59$ & $t=0.87$ \\
\hline \multicolumn{3}{|l|}{ Gender, $n(\%)$} & \multirow{3}{*}{$\chi^{2}=0.59$} \\
\hline Male & $8(33)$ & $11(44)$ & \\
\hline Female & $16(67)$ & $14(56)$ & \\
\hline \multicolumn{3}{|l|}{ Intellectual disability, $n$ (\%) } & \multirow{3}{*}{$\chi^{2}=1.64$} \\
\hline Mild (IQ score 50-70) & $12(50)$ & $8(32)$ & \\
\hline Moderate (IQ score 35-54) & $12(50)$ & $17(68)$ & \\
\hline \multicolumn{3}{|l|}{ IQ score } & \multirow{4}{*}{$\chi^{2}=0.49$} \\
\hline No IQ score available, $n(\%)$ & $14(58)$ & $17(68)$ & \\
\hline IQ score available, $n(\%)$ & $10(42)$ & $8(32)$ & \\
\hline IQ score, mean (s.d.) range & $54.00(6.00) 44-62$ & $55.63(7.41) 44-64$ & \\
\hline WIAT-II UK score, listening comprehension raw score: mean (s.d.) & $16.00(5.28)$ & $13.80(5.08)$ & $t=1.35$ \\
\hline \multicolumn{3}{|l|}{ Medication (for mood and anxiety disorders), $n$ (\%) } & \multirow{3}{*}{$\chi^{2}=0.50$} \\
\hline Receiving medication & $12(50)$ & $10(40)$ & \\
\hline Not receiving medication & $12(50)$ & $15(60)$ & \\
\hline \multicolumn{3}{|l|}{ Anxiety only or depression only, $n$ (\%) } & \multirow{3}{*}{$\chi^{2}=1.99$} \\
\hline Anxiety only & $13(65)$ & $10(43)$ & \\
\hline Depression only & $7(35)$ & $13(57)$ & \\
\hline \multicolumn{3}{|l|}{ Anxiety only and comorbid anxiety and depression, $n$ (\%) } & \multirow{3}{*}{$\chi^{2}=2.64$} \\
\hline Yes & $17(71)$ & $12(48)$ & \\
\hline No & $7(29)$ & $13(52)$ & \\
\hline \multicolumn{3}{|l|}{ Depression only and comorbid depression and anxiety, $n(\%)$} & \multirow{3}{*}{$\chi^{2}=0.99$} \\
\hline Yes & $11(46)$ & $15(60)$ & \\
\hline No & $13(54)$ & $10(40)$ & \\
\hline \multicolumn{3}{|l|}{ No other developmental disorder, $n(\%)$} & \multirow{3}{*}{$\chi^{2}=1.74$} \\
\hline No other developmental disorder & $10(41)$ & $6(24)$ & \\
\hline Other developmental disorder & $14(58)$ & $19(76)$ & \\
\hline \multicolumn{3}{|l|}{ Down syndrome, $n$ (\%) } & \multirow{3}{*}{$\chi^{2}=2.12$} \\
\hline Yes & $5(20)$ & $10(40)$ & \\
\hline No & $19(80)$ & $15(60)$ & \\
\hline \multicolumn{3}{|l|}{ No other psychological disorder, $n$ (\%) } & \multirow{3}{*}{$\chi^{2}=0.17$} \\
\hline No other psychological disorder & $14(58)$ & $16(64)$ & \\
\hline Other psychological disorder & $10(42)$ & $9(36)$ & \\
\hline \multicolumn{3}{|l|}{ GAS-ID } & \multirow{5}{*}{$t=0.28$} \\
\hline$n$ & 24 & 23 & \\
\hline Mean (s.d.) & $23.83(10.83)$ & $24.74(11.41)$ & \\
\hline Range, mean & Clinicall $^{\mathrm{C}}$ & Clinical $^{c}$ & \\
\hline Clinical range, $n$ & 21 & 18 & \\
\hline \multicolumn{3}{|l|}{ GDS-LD } & \\
\hline$n$ & 24 & 25 & \\
\hline Mean (s.d.) & $14.38(7.22)$ & $15.20(8.04)$ & $t=0.38$ \\
\hline Range, mean & Clinical $^{c}$ & Clinical $^{c}$ & \\
\hline Clinical range, $n$ & 14 & 13 & \\
\hline CORE-LD & & & \\
\hline$n$ & 23 & 23 & \\
\hline Mean (s.d.) & $11.57(5.92)$ & $12.17(7.40)$ & $t=0.31$ \\
\hline $\begin{array}{l}\text { CCBT, computerised cognitive-behavioural therapy; CORE, Clinical Outcomes } \\
\text { Disability; GDS-LD, Glasgow Depression Scale for people with a Learning Disa } \\
\text { a. } t \text {-value from independent } t \text {-test. } \\
\text { b. Chi-squared value from Pearson's } \chi^{2} \text {-tests; } \chi^{2} \text {-tests were not performed or } \\
\text { c. Scores of } 13 \text { or above fall within the clinical range on the GAS-ID and GDS }\end{array}$ & $\begin{array}{l}\text { uation - Learning Disability } \\
\text { cment as usual; WAIT-\| UK, } \\
\text { contained cells with an ex }\end{array}$ & $\begin{array}{l}\text {-ID, Glasgow Anxiety Sca } \\
\text { hsler Individual Achiever } \\
\text { ed count less than } 5 \text {. }\end{array}$ & $\begin{array}{l}\text { le with an Inte } \\
\text { Second UK Ed }\end{array}$ \\
\hline
\end{tabular}

wellness in people with an intellectual disability receiving psychotherapy. There are no clinical cut-off points available at present. The CORE-LD reports test-retest reliability of $r=0.64$ and has an internal consistency reliability of $\alpha=0.80$ and $\alpha=0.84$ in the present sample. ${ }^{18}$

\section{Sample size}

Using $\mathrm{G}^{*}$ Power $3.1^{19}$ a total of 54 participants is needed to power a $2 \times 3$ ANOVA to detect a medium effect size. In the current study, 52 participants powers a $2 \times 3$ ANOVA to detect an effect size of $f=0.33$, which is equivalent to $d=0.66^{20,21}$

\section{Randomisation}

Using the Microsoft Excel (2010) random list generator function, a random sequence of numbers was generated and applied to a total sample of 52 participants, assorting them into one list. The top 26 participants were assigned to the cCBT group and the bottom 26 participants were assigned to the TAU group. The primary researcher (P.C.) carried out this procedure.

\section{Allocation concealment}

As the primary researcher was responsible for the randomisation procedure, allocation concealment was not implemented in the present study. 


\section{Implementation}

The clinical psychologist was responsible for enrolling participants in the study from the service in which she was employed. The primary researcher was responsible for random assignment of participants to each group.

\section{Masking}

The present study did not use a masking procedure for participants or researchers. Owing to the natural design of this study to evaluate the effectiveness of a behavioural intervention, masking was not feasible as this was a non-drug trial. ${ }^{22}$

\section{Statistical methods}

Pre-treatment comparisons of demographic characteristics of each group were computed using Pearson's $\chi^{2}$ and independent $t$-tests for categorical and continuous data respectively. Independent $t$-tests were used to examine differences between the $\mathrm{CCBT}$ and TAU groups on primary and secondary outcome measures. Mixed between-within ANOVAs were used to examine the main effects for outcome variables. Following a significant interaction between groups, tests of simple effects were carried out to explore the nature of the interaction by examining the difference between groups within one level of one of the independent variables. Effect sizes expressing the degree to which $\mathrm{CCBT}$ group participants were better adjusted than those in the TAU group were calculated using Rosnow \& Rosenthal $^{23}$ and Cohen ${ }^{20}$ criteria. Reliable Change Indices (RCIs) ${ }^{24}$ were carried out to examine clinical recovery rates.

To treat missing data, mean substitution was used for cases which had no more than $10 \%$ of data missing on a scale at a given time point. As there were 27 items on the GAS-ID, ${ }^{16}$ mean substitution was used for cases with 3 or fewer items missing on that scale.

\section{Results}

There were two components in this analysis. First, to establish whether the CCBT intervention led to statistically significant improvement on symptom measures of anxiety, depression and psychological wellness from pre- to post-treatment to 3-month follow-up. Second, to evaluate the extent to which the cCBT intervention led to clinically significant change, clinical recovery rates of the cCBT and TAU groups on the primary outcome measure of anxiety were compared.

\section{Participant flow}

A CONSORT diagram presenting the randomisation process and participant flow through the study can be seen in Fig. 1 (online Appendix DS1 shows CONSORT inclusion criteria). As a per-protocol analysis was used, participants who were absent from data collection at post-treatment or follow-up were excluded from the final analysis.

One participant who was randomly assigned to the cCBT group was subsequently excluded from receiving the intervention as the participant had experienced a relapse in mental health to a degree to which they were unable to avail of psychological therapy. Of the remaining 25 cCBT group participants, there was no drop-out at post-treatment. One participant from the cCBT group did not provide any data at follow-up as they were unable to complete the outcome measures in a single sitting at the specific time point.
Of the 26 TAU participants, there was no-drop out at posttreatment. One participant did not provide any data at follow-up. This participant was uncontactable; thus, they had dropped out of the study and were not included in the final analysis.

Regarding missing data at pre-treatment: 2 TAU participants were missing more than $10 \%$ on the GAS-ID, and 1 cCBT participant and 2 TAU participants were missing more than $10 \%$ on the CORE-LD. At post-treatment: 1 cCBT participant and 3 TAU participants were missing more than $10 \%$ on the CORE-LD. At follow-up: 1 TAU participant was missing more than $10 \%$ on the CORE-LD.

\section{Recruitment}

Eligible participants were recruited in February 2015. Participants in the CCBT group attended their data collection setting (onsite in the main centre, within their onsite residence, or offsite in community day-service centres) at pre-treatment (intervention week 1), for 7 consecutive weeks to post-treatment (intervention week 7), and at 3-month follow-up from March 2015 to September 2016. Participants in the TAU group attended their data collection setting at pre-treatment, post-treatment, and 3-month follow-up from March 2015 to September 2016.

\section{Outcomes and estimation}

Six $2 \times 3$ (group $\times$ time) mixed between-within ANOVAs were used to investigate statistically significant improvements in symptom scores on GAS-ID total anxiety, the three GAS-ID subscales, GDS-LD total depression, and CORE-LD total psychological wellness. Table 2 summarises these analyses. Mauchly's test of sphericity determined that assumption for sphericity was met in the ANOVAs of the primary outcome measures of anxiety (GAS-ID and its subscales) and depression. The assumption for sphericity was violated in the ANOVA for the CORE-LD. Based on the observed epsilon values of Mauchly's test of sphericity, the Huynh-Feldt value was used from the ANOVA table. Significant group $\times$ time interactions were observed on the total score of primary outcome measure of anxiety (GAS-ID) and both its Worries and Physiological Symptoms subscales. Tests of simple effects were carried out to compare means between both groups and across time to identify the differences that led to these statistically significant interactions in these ANOVAs. Regarding all three interactions, tests of simple effects revealed that pre-treatment TAU group scores remained constant, whereas CCBT group scores improved significantly from pre- to post-treatment and from post-treatment to follow-up. These interactions illustrating a significant reduction in the CCBT group's total anxiety symptoms, worries and physiological symptoms across time in comparison with the TAU group are graphed in online Fig. DS2. No significant between-groups interaction was observed on the GAS-ID Specific Fears subscale.

No significant between-groups interactions were observed for the primary outcome measure of depression (GDS-LD) or the secondary outcome measure of psychological wellness (CORE-LD).

Effect sizes were computed using the means and standard deviations of both the cCBT and TAU groups at post-treatment and follow-up as presented in Table 3. Effect sizes were interpreted using Cohen's (1988) criteria $^{20}$ where effect sizes of $d=0.2$ are considered small, $d=0.5$ are considered medium, and $d=0.8$ are considered large. Considering the statistically significant change in the cCBT group's total anxiety symptoms (mean GAS-ID scores), it was determined that this was a medium effect size $(d=0.67)$ at post-treatment and a large effect size $(d=1.10)$ at follow-up. Effect sizes expressing the degree to which participants 


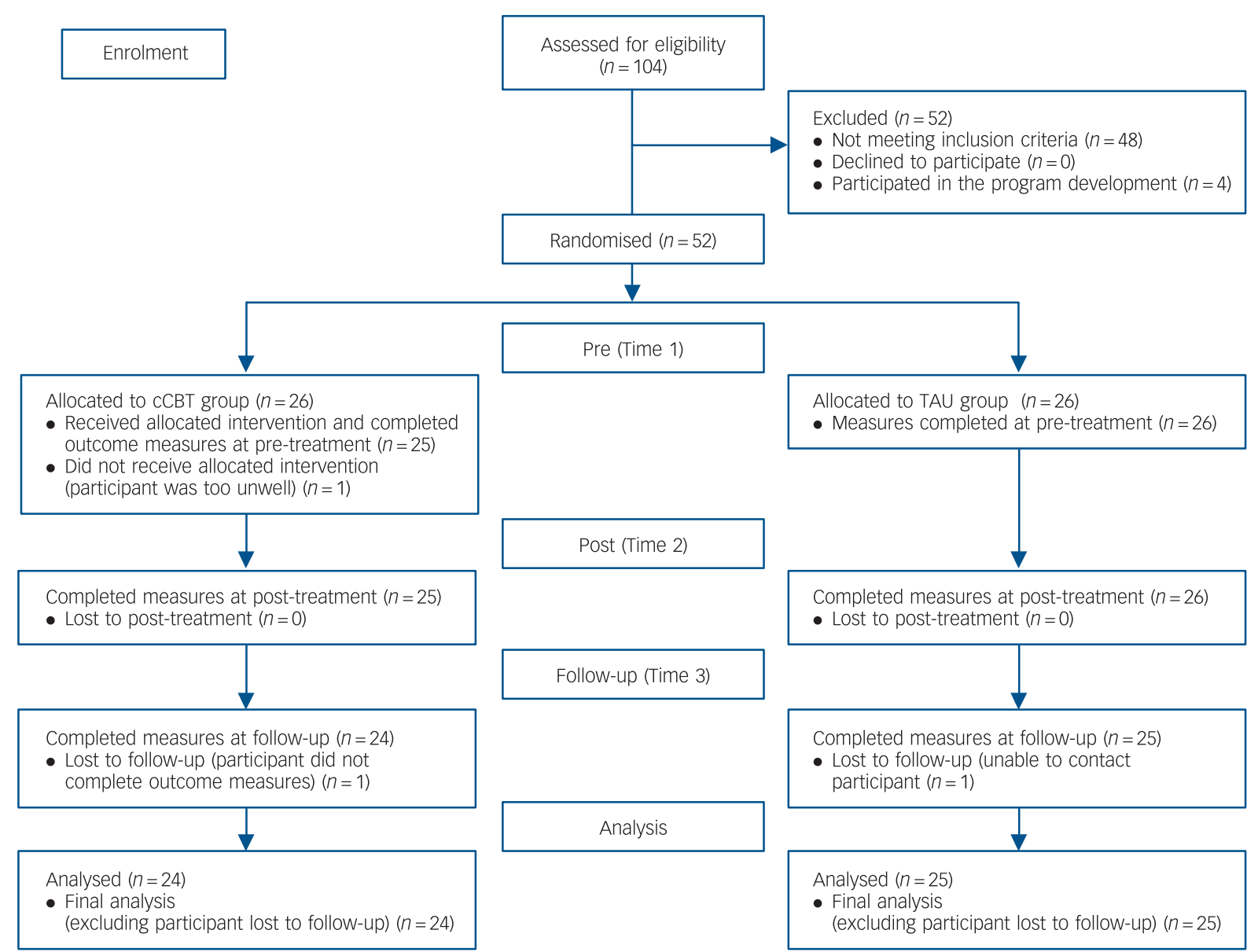

Fig. 1 CONSORT participant flow diagram through the study. CCBT, computerised cognitive-behavioural therapy; TAU, treatment as usual.

in the CCBT group were better adjusted on total anxiety symptoms (mean GAS-ID scores) than those in the TAU group can be considered medium $(d=-0.51)$ at post-treatment to large $(d=-1.09)$ at follow-up.

\section{Clinically significant change}

Clinical recovery refers to the extent to which therapy led to a clinically meaningful improvement in an individual's life, the extent to which therapy moves someone outside the range of the dysfunctional population or within the range of the functional population. ${ }^{24}$ To investigate whether there was a clinically significant change in anxiety symptoms in the CCBT group in comparison to the TAU group, the Jacobson-Truax method was used to calculate an RCI. Cases were classified as in remission if they moved from the clinical ( 13 and above) to non-clinical range (12 and below) from pre- to post-treatment or follow-up on the primary outcome measure of anxiety symptoms (GAS-ID), and also had an RCI value greater than 1.96 as stated by Jacobson \& Truax. ${ }^{24}$ The RCI is calculated for each participant taking into account the psychometric properties of the scale and their mean scores between specific time points. Table 4 reports remission rates in the CCBT and TAU groups. Chi-squared analyses revealed a significant difference between groups regarding cases classified as being in remission and non-improvers, with the CCBT group having significantly greater anxiety remission rates than that TAU group.

\section{Harms}

Based on the outcome analyses no harmful or negative effects were observed during this study.

\section{Discussion}

The aim of this study was to evaluate the utility of a prototype CBT computer game, Pesky Gnats: The Feel Good Island. This intervention was observed to produce clinically significant change in anxiety symptoms in comparison with psychiatric TAU in adults who have an intellectual disability. The intervention had a medium-sized effect on anxiety symptoms post-intervention and a large-sized effect after a 3-month follow-up period. Furthermore, the intervention facilitated clinical recovery of anxiety (i.e. reliable change), whereby it led to a clinically meaningful improvement for almost $40 \%$ of people 3 months after completing the intervention. This finding is both positive and novel as the literature to date has not established an evidence base for CBT interventions for the treatment of anxiety in this population. ${ }^{3,25}$

When considering the differences between Pesky Gnats: The Feel Good Island and existing transdiagnostic CBT interventions, the incorporation of two novel components - technology and mindfulness relaxation exercises - may play a role in our positive findings for anxiety symptoms. Technology assisting mental health intervention is a novel approach with this population. The Pesky gNATs model is one such approach assisting therapists in the delivery of an evidence-based mental health intervention while maintaining all the characteristics of a good therapeutic relationship. ${ }^{26}$ CBT emphasises active participation of both the therapist and client as a key element in establishing a collaborative therapeutic relationship. ${ }^{27}$ This collaborative relationship can then be used to elicit the benefits of Vygotsky's ${ }^{28}$ concept of a zone of proximal development to support an adult with an intellectual 

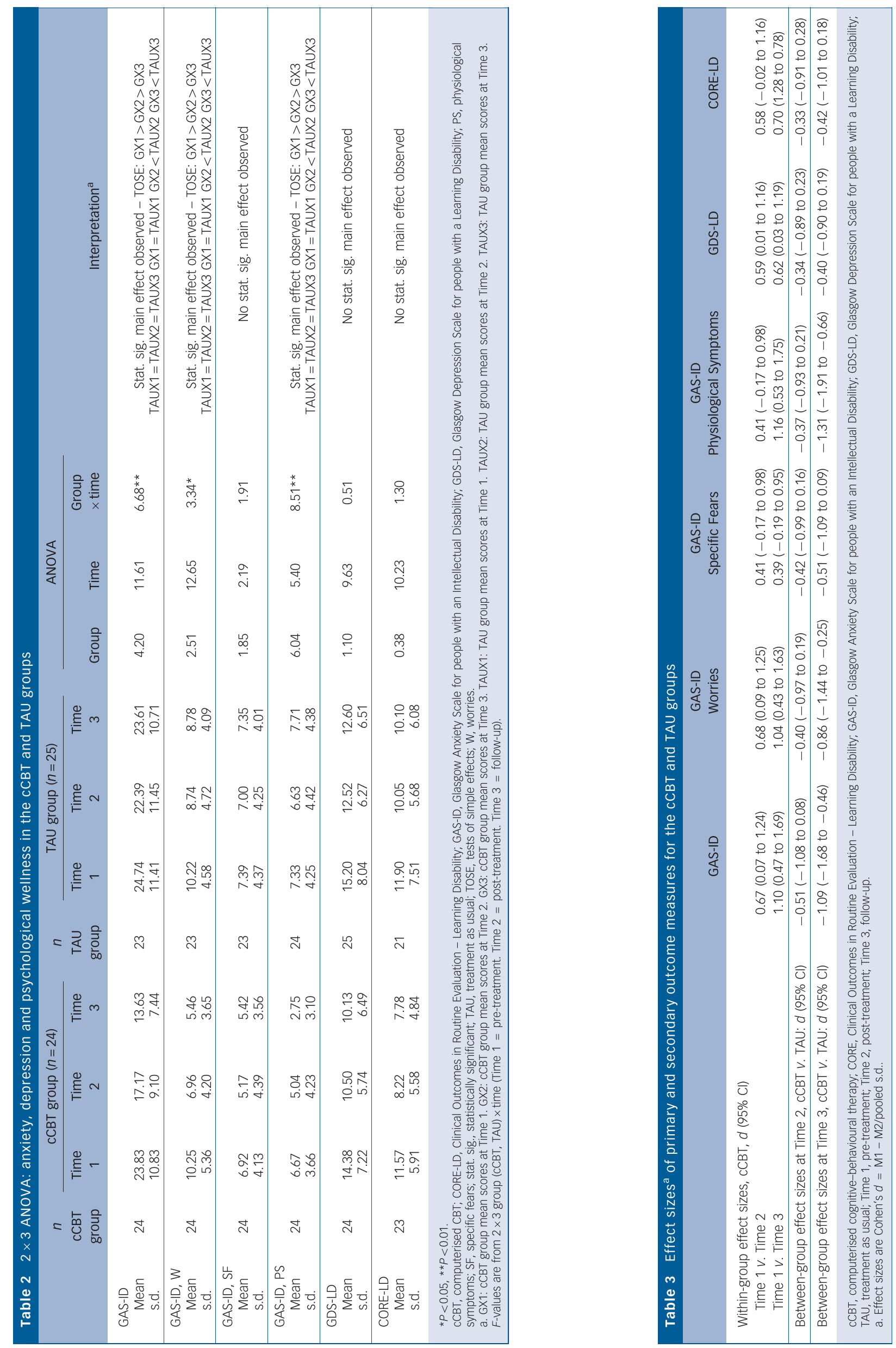


\begin{tabular}{|c|c|c|c|c|c|}
\hline & $\begin{array}{l}\text { cCBT group } \\
\quad(n=24)\end{array}$ & $\begin{array}{l}\text { TAU group } \\
(n=23)\end{array}$ & Chi-squared $^{\text {a }}$ & $P$ & Group differences $^{b}$ \\
\hline $\begin{array}{l}\text { Time } 2 \text { (post-treatment), } n \text { (\%) } \\
\text { In remission }{ }^{c} \\
\text { Non-improvers }\end{array}$ & $\begin{array}{r}7(29.20) \\
17(70.80)\end{array}$ & $\begin{array}{c}1(4.30) \\
22(95.70)\end{array}$ & 5.12 & 0.02 & $1>2$ \\
\hline $\begin{array}{l}\text { Time } 3 \text { (follow-up), } n \text { (\%) } \\
\text { In remissionc } \\
\text { Non-improvers }\end{array}$ & $\begin{array}{r}9(37.50) \\
15(62.50)\end{array}$ & $\begin{array}{r}2(8.70) \\
21(91.30)\end{array}$ & 5.44 & 0.02 & $1>2$ \\
\hline \multicolumn{6}{|c|}{$\begin{array}{l}\text { CCBT, computerised CBT; TAU, treatment as usual. } \\
\text { a. Both Chi-Squared tests contained cells with an expected count less than } 5 \text {. } \\
\text { b. Group differences: } 1>2=\text { group } 1 \text { (CCBT) had greater recovery rates than group } 2 \text { (TAU). } \\
\text { c. Cases were classified as in remission if they moved from the clinical ( } 13 \text { and above) to non-clinical range (12 and below) from Time } 1 \text { (pre-treatment) to Time } 2 \text { or Time } 3 \text { on the } \\
\text { Glasgow Anxiety Scale for people with an Intellectual Disability, and if they also had a Reliable Change Index value greater than } 1.96 \text {. }\end{array}$} \\
\hline
\end{tabular}

disability to scaffold their thinking about their thoughts, feelings and behaviours in a way that is to their benefit. The use of a computer-assisted program has the potential to help an adult with an intellectual disability to achieve more in collaboration with their therapist than they can alone or without the aid of technology. In this way, the Pesky Gnats: Feel Good Island model provides reliable supports in the form of a computer game and accompanying workbook that add to the benefits of a good therapist-client therapeutic relationship.

The intervention did not demonstrate a significant reduction in depression symptoms. This finding appears in contrast to existing literature which has recently begun to document an evidence-base for CBT in the treatment of depression in this population. ${ }^{3,4,25}$ It may be that the contents of the program were not a good match for participants with depression in this study. Alternatively, a smaller number of participants had a diagnosis of depression in comparison with those who had a diagnosis of anxiety. The mean score on the depression scale was just above the clinical cut-off at pre-treatment and was in the normal range at post-treatment and follow-up for both groups. Therefore, a possible limitation of the present study was the relatively low levels of depression among all participants and the need for greater statistical power to detect any differences between groups should they exist.

Although the finding that Pesky Gnats: The Feel Good Island produced a large-sized effect for the treatment of anxiety is largely positive, it highlights the lack of change experienced by participants receiving usual psychiatric treatment. ${ }^{3}$

\section{Strengths and limitations of the study}

The inclusion of a 3-month follow-up in the design of the present study provided an acceptable indication of the long-term effects of the intervention in a group of people for whom sustained learning is particularly difficult. The use of the $\mathrm{RCI}^{24}$ is a strength of the current study as it offers an indication of the magnitude of impact of the intervention in terms of clinically meaningful improvement in a person's life.

Limitations of the present study are that (a) just one organisation took part and (b) it is not sufficiently powered to carry out further analysis on whether participant characteristics such as age, gender, intellectual disability diagnosis, listening comprehension ability, or dual developmental diagnosis such as autism spectrum disorder or Down syndrome impact engagement with this intervention. Future research should aim to obtain larger multisite trials to explore these factors further. An additional limitation is that an intention-to-treat analysis was not used. However, given that 49 out of 52 cases were analysed in the present study, we would anticipate that similar results would be obtained from an intention-to-treat analysis.

\section{Clinical implications}

While acknowledging there is still much work to be done in this area, the present study makes a promising contribution to the development of appropriate evidence-based psychological interventions to meet the mental health needs of adults who have a mild to moderate intellectual disability. To the authors' knowledge Pesky Gnats: The Feel Good Island is the first application of CCBT in this population and having demonstrated efficacy in reducing anxiety symptoms it appears to be an effective treatment option. Studies like this contribute to breaking down the barriers that prevent adults with an intellectual disability accessing effective user-friendly and destigmatising mental health technologies. The other strand of the research reported here explores the experiences of the adults who played the prototype game to provide guidance for developers of future technologyassisted CBT programs for this population (details available from the author on request).

Patricia Cooney, BA, School of Psychology, University College Dublin; Catherine Jackman, MPsychSc, Cheeverstown House, Dublin; David Coyle, PhD, School of Computer Science, University College Dublin; Gary O'Reilly, PhD, School of Psychology, University College Dublin, Ireland

Correspondence: Patricia Cooney, School of Psychology, Newman Building, University College Dublin, Belfield, Dublin 4, Ireland. Email: patricia.cooney@ucdconnect.ie

First received 16 Jan 2017, final revision 3 Mar 2017, accepted 17 Mar 2017

\section{References}

1 Cooper S-A, Smiley E, Morrison J, Williamson A, Allan L. Mental ill-health in adults with intellectual disabilities: prevalence and associated factors. $\mathrm{Br} J$ Psychiatry 2007; 190: 27-35.

2 Kessler RC, Aguilar-Gaxiola S, Alonso J, Chatterii S, Lee S, Ormel J, et al. The global burden of mental disorders: an update from the WHO World Mental Health (WMH) surveys. Epidemiol Psichiatr Soc 2009; 18: 23-33.

3 Koslowski N, Klein K, Arnold K, Kösters M, Schützwohl M, Salize HJ, et al. Effectiveness of interventions for adults with mild to moderate intellectual disabilities and mental health problems: systematic review and metaanalysis. Br J Psychiatry 2016; 209: 469-74

4 National Institute for Health and Care Excellence. Mental Health Problems in People with Learning Disabilities: Prevention, Assessment and Management (NICE guideline NG54). NICE, 2016 (https://www.nice.org.uk/guidance/ng54).

5 Willner P, Rose J, Jahoda A, Kroese BS, Felce D, Cohen D, et al. Group-based cognitive-behavioural anger management for people with mild to moderate intellectual disabilities: cluster randomised controlled trial. Br J Psychiatry 2013; 203: 288-96.

6 Hassiotis A, Serfaty M, Azam K, Strydom A, Blizard R, Romeo R, et al. Manualised individual cognitive behavioural therapy for mood disorders in people with mild to moderate intellectual disability: a feasibility randomised controlled trial. J Affect Disord 2013; 151: 186-95.

7 Lindsay WR, Tinsley S, Beail N, Hastings RP, Jahoda A, Taylor JL, et al. A preliminary controlled trial of a trans-diagnostic programme for cognitive 
behaviour therapy with adults with intellectual disability. J Intellect Disabil Res 2015; 59: 360-9.

8 National Institute for Health and Care Excellence. Depression in Adults: Recognition and Management (Update) (NICE Clinical Guideline 90). NICE, 2016 (https://www.nice.org.uk/CG90).

9 den Brok WLJE, Sterkenburg PS. Self-controlled technologies to support skill attainment in persons with an autism spectrum disorder and/or an intellectual disability: a systematic literature review. Disabil Rehabil Assist Technol 2015; 10: 1-10.

10 Vereenooghe L, Gega L, Reynolds S, Langdon PE. Using computers to teach people with intellectual disabilities to perform some of the tasks used within cognitive behavioural therapy: a randomised experiment. Behav Res Ther 2016; 76: 13-23.

11 Tanis ES, Palmer S, Wehmeyer M, Davies DK, Stock SE, Lobb K, et al. Selfreport computer-based survey of technology use by people with intellectual and developmental disabilities. Intellect Dev Disabil 2012; 50: 53-68.

12 O'Reilly G, Cooney P, Jackman C, Coyle D. Pesky Gnats: The Feel Food Island A CBT Computer Game to Assist Adults who have an Intellectual Disability with Anxiety or Depression. Handaxe $\mathrm{CIC}, 2015$.

13 O'Reilly G, Coyle D. Pesky gNATs. A CBT Computer Game to Assist Young People with Anxiety or Low Mood. Handaxe CIC, 2015

14 O'Reilly G, Coyle D. The Pesky gNATs App. Handaxe CIC, 2015.

15 Beck AT, Haigh EA. Advances in cognitive theory and therapy: the generic cognitive model. Annu Rev Clin Psychol 2014; 10: 1-24.

16 Mindham J, Espie C. Glasgow Anxiety Scale for people with an Intellectual Disability (GAS-ID): development and psychometric properties of a new measure for use with people with mild intellectual disability. J Intellect Disabil Res 2003; 47: 22-30.

17 Cuthill FM, Espie CA, Cooper S-A. Development and psychometric properties of the Glasgow Depression Scale for people with a Learning Disability Individual and carer supplement versions. Br J Psychiatry 2003; 182: 347-53.
18 Brooks M, Davies S, Twigg E. A measure for feelings - using inclusive research to develop a tool for evaluating psychological therapy (Clinical Outcomes in Routine Evaluation-Learning Disability). Br J Learn Disabil 2013; 41: 320-9.

19 Faul F, Erdfelder E, Buchner A, Lang A-G. Statistical power analyses using G* Power 3.1: Tests for correlation and regression analyses. Behav Res Methods 2009; 41: 1149-60.

20 Cohen J. Statistical Power Analysis for the Behavioral Sciences (2nd edn). Erlbaum, 1988.

21 Carr A. What Works with Children, Adolescents, and Adults? A Review of Research on the Effectiveness of Psychotherapy. Routledge, 2009.

22 Higgins JP, Altman DG, Gøtzsche $P C$, Jüni $P$, Moher $D$, Oxman $A D$, et al. The Cochrane Collaboration's tool for assessing risk of bias in randomised trials. BMJ 2011; 343: d5928.

23 Rosnow RL, Rosenthal R. Computing contrasts, effect sizes, and counternulls on other people's published data: general procedures for research consumers. Psychol Methods 1996; 1: 331

24 Jacobson NS, Truax P. Clinical significance: a statistical approach to defining meaningful change in psychotherapy research. J Consult Clin Psychol 1991; 59: 12 .

25 Vereenooghe L, Langdon PE. Psychological therapies for people with intellectual disabilities: a systematic review and meta-analysis. Res Dev Disabil 2013; 34: 4085-102

26 O'Reilly G, Coyle D, Tunney C. Even Buddhist monks use a gong: a mindfulness skills programme for young people delivered through the "Mindful Gnats" computer game and App. Int J Game-Based Learning (IJGBL) 2016; 6: 39-51.

27 Beck JS. Cognitive Behavior Therapy: Basics and Beyond. Guilford Press, 2011.

28 Vygotsky LS. Mind in Society: The Development of Higher Psychological Processes. Harvard University Press, 1978.

\section{poems by doctors}

\section{Noise in her Mind}

\section{Kai Michael Neumann}

I know her so well she came long ago to visit my being we share beauty and bedlam boudoir and kindest of spirits

She walks like a cat woman hears like a bat feeds birds quietly she soothes thunder's volcano howls with the wolves

Klimt's Kiss Vermeer's Girl With Pearl Earring Botticelli's Venus Degas's

Dancer In Repose Fedro's Much Better With Age she

absorbs pastel and paint strokes symphonies on canvas

so many facets big bangs and roaring giggling convulsions

Sometimes Woman Laughing from Goya's dark paintings

a Broken Column like Kahlo's when some Munch's Scream

invades and infests silence deep inside her beautiful scull

Voices shout from profuse directions focus condense and refract confront tease torment disagree and interfere with calm serene serenades when a few notes tender chords and accord vanish into cacophonies misleading the song

I know her so well admire her resilience and how she copes with the noise the disruption and the ballet of so many different views pandemonious clangour onslaught of turmoil as she appears calm on the surface orchestrates quiet composure

How she holds it all together I cannot really fathom with all that jumbling distance so close and yet so far from my innocent mind

My Venus Of Milo has taught me so much most of it all and over above that imperfection is a wonderful gift and very complete

(c) Kai Michael Neumann. Reproduced with permission. 